\title{
Materials Classification using Sparse Gray-Scale Bidirectional Reflectance Measurements
}

\author{
Jiř́ Filip ${ }^{1}$, Petr Somol ${ }^{1,2}$ \\ ${ }^{1}$ Institute of Information Theory and Automation of the CAS, Czech Republic \\ ${ }^{2}$ Faculty of Management, Prague University of Economics, Czech Republic
}

\begin{abstract}
Material recognition applications use typically color texturebased features; however, the underlying measurements are in several application fields unavailable or too expensive (e.g., due to a limited resolution in remote sensing). Therefore, bidirectional reflectance measurements are used, i.e., dependent on both illumination and viewing directions. But even measurement of such BRDF data is very time- and resources-demanding. In this paper we use dependency-aware feature selection method to identify very sparse set of the most discriminative bidirectional reflectance samples that can reliably distinguish between three types of materials from BRDF database - fabric, wood, and leather. We conclude that ten gray-scale samples primarily at high illumination and viewing elevations are sufficient to identify type of material with accuracy over $96 \%$. We analyze estimated placement of the bidirectional samples for discrimination between different types of materials. The stability of such directional samples is very high as was verified by an additional leave-one-out classification experiment. We consider this work a step towards automatic method of material classification based on several reflectance measurements only.
\end{abstract}

Keywords: BRDF, material, classification, feature selection

\section{Introduction}

The real-world is made of countless varieties of materials having a wide range of appearance. Their automatic segmentation and classification is vital in applications where the task is quick identification of material type, based purely on its directional reflectance, i.e., without considering its surface texture and color. An automatic material type detection based purely on several reflectance measurements is relevant research task in many research fields ranging from remote sensing to paint industry, food inspection, or recycling. Especially in remote sensing a constrained set of illumination and viewing directions plays important role in significant increase of recognition accuracy [9].

Although it might seem that using reflectance only, instead of texture, might solve a data dimensionality problem, we still face a vast number of possible combinations of illumination and viewing directions. Therefore, identification those of them that have the best performance in distinguishing between different material categories is a challenging research task. 
Such a bidirectional reflectance behavior can be formalized by means of Bidirectional Reflectance Distribution Function (BRDF) [7] comprising differential reflected radiance $d L$ for incident irradiance $d E$

$$
B\left(\theta_{i}, \varphi_{i}, \theta_{v}, \varphi_{v}\right)=\frac{d L\left(\theta_{v}, \varphi_{v}\right)}{d E\left(\theta_{i}, \varphi_{i}\right)}=\frac{d L\left(\theta_{v}, \varphi_{v}\right)}{L\left(\theta_{i}, \varphi_{i}\right) \cos \theta_{i} d \omega_{i}} .
$$

BRDF is a four dimensional function of incoming $\left[\theta_{i}, \varphi_{i}\right]$ and outgoing $\left[\theta_{v}, \varphi_{v}\right]$ spherical angles as shown in Fig. 1-left. Helmholtz reciprocity states that illumination and viewing directions can be swapped without any effect on the BRDF value.
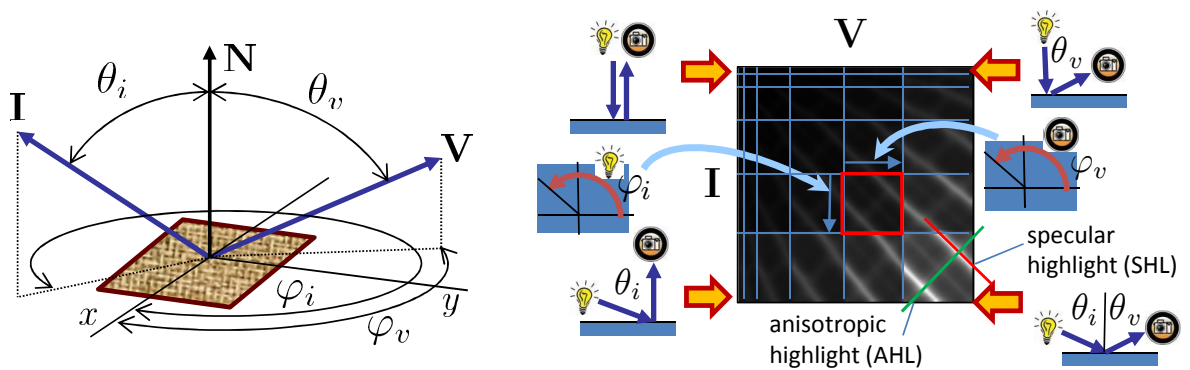

Fig. 1. A four-dimensional BRDF angular parameterization (left) and its unfolding into 2D image (right), depicting locations of specular (SHL) and anisotropic (AHL) highlights. The unfolded 2D image belongs to mean gray-scale BRDF of all tested materials. Each block (shown in red frame) depicts azimuthally-dependent $\left(\varphi_{i} / \varphi_{v}\right)$ reflectance behavior for fixed elevation angles $\theta_{i} / \theta_{v}$.

BRDF measurements are typically fitted by analytical models achieving a compact parametric representation, missing directional data interpolation / extrapolation, as well as removal of outliers resulting from the measurement process [4]. However, reliable fitting of BRDF models to the measured reflectance values require sufficient coverage of illumination and viewing directions over the measured material. Such measurement is both time- and space-demanding and requires specialized and often expensive measurement gantry [4].

In this paper we attempt to analyze properties of three material categories using linear factorization and feature selection. Our goal is to identify an extremely compact and discriminative set of illumination/view directions combinations, that can be rapidly and inexpensively measured, and finally used for material type classification purposes. Therefore, identification of such compact set of bidirectional features reduces not only data measurement time and costs, but also computational requirements of training and classification steps of discriminative model. Therefore, the main contribution of this paper is employing feature selection (FS) technique to identify sparse directional reflectance samples appropriate for reliable material classification. Our method relies on a typical unique behavior of different types of materials, therefore, we run the FS on 
extensive BRDF database specifically focusing on distinguishing between three types of materials: fabric, leather, and wood.

The paper is structured as follows. Section 2 relates our research to prior work in the field. Section 3 introduces BRDF data used and analyses data within individual material categories. Section 4 proposes the method of detection of the most discriminative directional samples. Section 5 outlines the achieved results while Section 6 concludes the paper.

\section{Prior Work}

An automatic material type classification based on its reflectance, i.e., without need of surface texture analysis, is vital in fields of remote sensing, paint industry, food inspection, material science, recycling etc.

Prior material segmentation/classification approaches in general rely also on a very limited set of directional measurements constrained by an intended application. Known approaches use often spectral illumination, which is distributed into variable illumination pattern over hemisphere. Common application scenario is also pixel-wise segmentation of real-world object to different materials [14], [12]. Gu et al. [3] introduced spectral coded illumination by discriminative patterns, and constructed an color LED-based measurement dome for material classification. Method of material classification based on illumination using variable illumination patterns combined with random forest classifier to identify optimal illumination is presented in [5]. A recent material classification approach [15] was based on using extended full bidirectional texture measurements as a training dataset. The classification task was to recognize a correct material given a single image of its surface in arbitrary illumination/viewing conditions.

We propose a feature selection method determining an extremely sparse set of discriminative directional measurement that allows very accurate discrimination of different types of materials. Contrary to the works above and for the sake of computational simplicity we do not take into consideration any textural or spectral information.

Remote sensing applications rely on a constrained set of viewing directions (airborne or satellite imagery) and illumination directions (directional illumination of the Sun combined with diffuse atmospheric illumination). The resulted sparse measurements are typically compensated for atmospheric radiative transfer and fitted by an analytical BRDF model. A typical goal of remote sensing application is detection of presence or classification of vegetation types [9] or urban areas [6] based on its spectral and directional reflectance. Qi et al. [8] fitted leaf and vegetation directional measurements using BRDF models. Schaaf et al. [10] process data from NASAs Terra satellite and the MODerate Resolution Imaging Spectroradiometer to provide BRDF and albedo of Earth surface. Several case studies of bidirectional reflectance measurements in remote sensing applications are reviewed in [11].

Our results can be extended to application areas where the directional measurements are expensive or constrained as for instance in the case of remote 
sensing. Therefore, we use the same BRDF representation and test our method on a BRDF dataset containing considerable information on the illumination- and view-dependent behavior of substantial collection of various materials. By analyzing this entire collection of bidirectional samples using feature selection techniques, we aim to obtain the most discriminative set of bidirectional samples.

\section{Test Data Analysis}

\subsection{Test Dataset}

We used UTIA BRDF Database ${ }^{3}$ [2] containing 150 anisotropic BRDF measurements. To enlarge its descriptive abilities we added another 67 BRDF anisotropic measurements obtained by averaging of BTFs with rough surface structure. We use the original resolution of BRDF measurements $81 \times 81$ illumination and viewing directions resulting in 6561 combination of incoming and outgoing directions. This number can be due to BRDF reciprocity reduced to 3321 directions. Finally, we grouped the measured BRDFs into three main categories: fabric (146 materials), leather (16 materials), and wood (19 materials). The remaining types of materials were not represented by a sufficient number of instances to capture their typical behavior reliably (carpet 6 , plastic 6 , tile 4 , paper 3 , wallpaper 3 , plaster 2, paint 2, etc.). Due to the fact, that some of the materials have many different color variants with similar luminance behavior, individual BRDFs were converted to the luminance only, neglecting the spectral information. Moreover, as anisotropic behavior in measured BRDF depends on initial positioning of the measured sample, all BRDFs were aligned according a location of their the most intensive anisotropic highlight in azimuthal space [2]. Any reasonable computational comparison of different anisotropic BRDFs would be impossible without this step as the anisotropic highlight would be located in the azimuthal space arbitrarily.

\subsection{Linear Factorization}

First, we analyzed the main visual features of individual types of materials using principal component analysis (PCA). The BRDFs of each category were reshaped into column vectors and used to form a matrix that was after normalization decomposed by means of PCA. As a result we obtained principal components of the same size as original BRDF image, and principal values denoting contribution of individual principal components. Fig. 3 shows mean and standard deviation images as well as the first three principal components (PC) for the tested categories of materials. Mean standard deviation values as well as energies of individual components suggests that the highest variability is within categories fabric and leather. While leather retains the most energy in its first component, the energy distribution within fabric is more uniform. This is due to a higher variance within this category given by much higher number of recorded

\footnotetext{
${ }^{3}$ http://btf.utia.cas.cz
} 


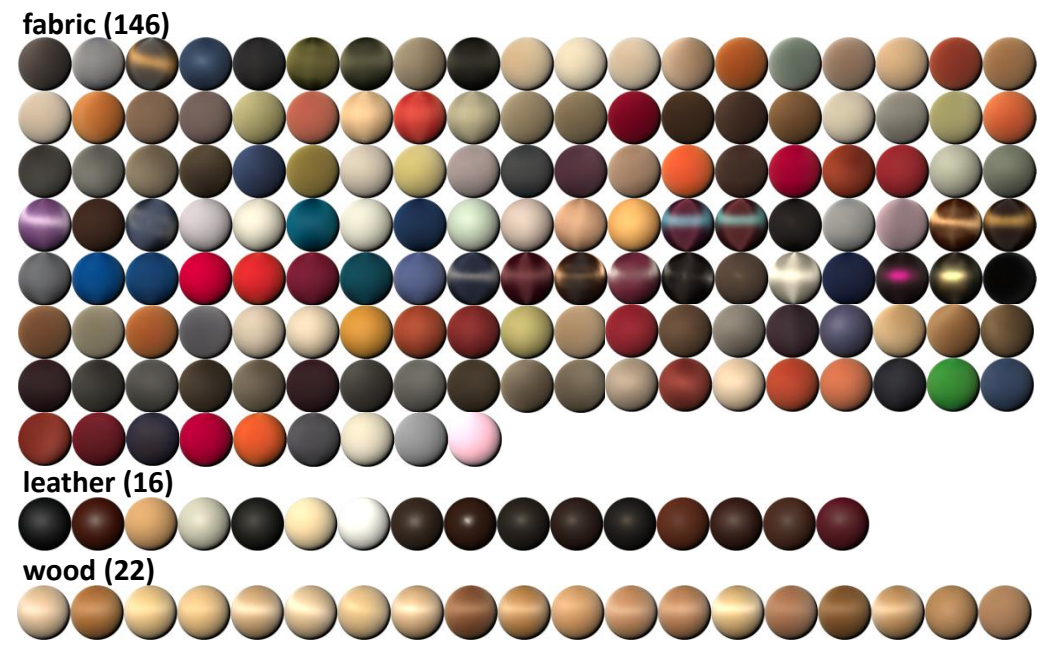

Fig. 2. BRDFs of three material categories used in our analysis.

materials, and also due to different types of anisotropy presented (as shown in principal images). Although wood category also exhibits strong anisotropy, the energy is shifted towards first components. This is caused by a more accurate anisotropic highlights alignment and similarly shaped highlights across materials in this category.

\section{Dependency-Aware Feature Selection}

The above statistical differences between the three material categories inspired us as to the possibility there might be certain sparse angular features bearing all information needed for their discrimination. To this end, we employed a combination of ranking and randomization feature selection method called Dependency-Aware Feature (DAF) ranking [13],[1]. It evaluates features' contributions in a sequence of randomly generated feature subsets. The method has shown promise of selecting features reliably, even in settings where standard feature techniques fail due to problem's complexity or over-fitting issues, and where individual feature ranking results are unsatisfactory.

Denoting $F$ the set of all features $F=\left\{f_{1}, f_{2}, \ldots, f_{N}\right\}$ we assume that for each subset of features $S \subset F$ a feature selection criterion $J(\cdot)$ can be used as a measure of quality of $S$. We assume the criterion $J(\cdot)$ to be bounded by $[0,1]$. In our case we will use estimates of classification accuracy which fulfills this property.

The starting point of dependency-aware feature ranking is a randomly generated sequence of feature subsets to be denoted probe subsets $\mathbf{S}=\left\{S_{1}, S_{2}, \ldots, S_{K}\right\}$, $S_{j} \subset F, j=1,2, \ldots, K$, where each subset is evaluated by the criterion function $J(\cdot)$. 


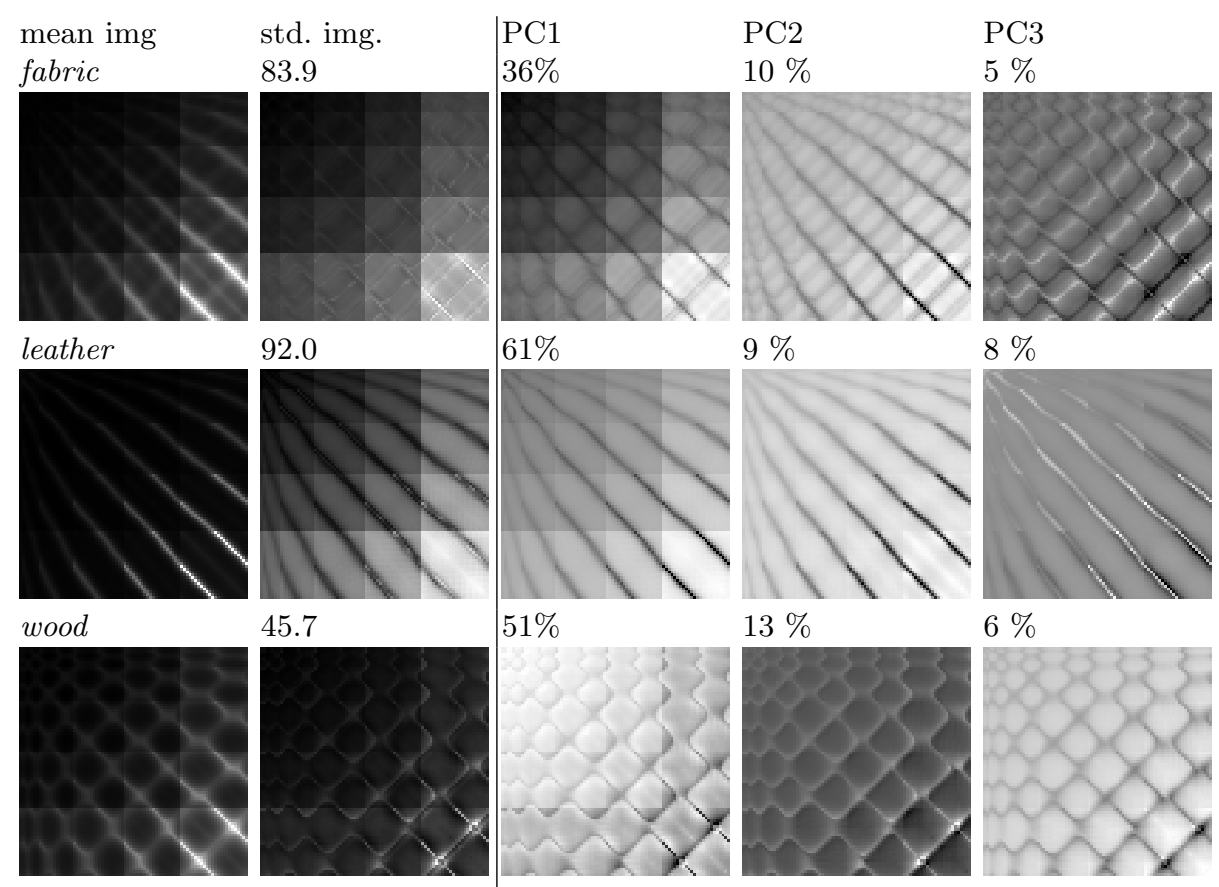

Fig. 3. PCA of three tested material categories BRDFs unfolded to 2D images (see Fig. 1-right). Each row contains mean image, standard deviation image (with mean std. value), and the first three principal components with their energy contributions.

Given a sufficiently large sequence of feature subsets $\mathbf{S}$, we can utilize the information contained in the criterion values $J\left(S_{1}\right), J\left(S_{2}\right), \ldots, J\left(S_{K}\right)$ to assess how each feature adds to the criterion value. Therefore, we compare the quality of probe subsets containing $f$ with the quality of probe subsets not including $f$.

We compute the mean quality $\mu_{f}$ of subsets $S \in \mathbf{S}$ containing the considered feature

$$
\mu_{f}=\frac{1}{\left|\mathbf{S}_{f}\right|} \sum_{S \in \mathbf{S}_{f}} J(S), \quad \mathbf{S}_{f}=\{S \in \mathbf{S}: f \in S\}
$$

and the mean quality $\bar{\mu}_{f}$ of subsets $S \in \mathbf{S}$ not containing the considered feature $f:$

$$
\bar{\mu}_{f}=\frac{1}{\left|\overline{\mathbf{S}}_{f}\right|} \sum_{S \in \overline{\mathbf{S}}_{f}} J(S), \quad \overline{\mathbf{S}}_{f}=\{S \in \mathbf{S}: f \notin S\}
$$

with the aim to use the difference of both values as a criterion for ranking the features:

$$
D A F(f)=\mu_{f}-\bar{\mu}_{f}, \quad f \in F .
$$

The DAF evaluates a contribution of each feature to a given discrimination task by a single weight value. The higher is its value the more important it is. More details can be found in [13]. 


\subsection{DAF Configuration Within the Experiment}

We used DAF method implemented in the FST3 library ${ }^{4}$ to compute ranking of measured directions according to their contribution to material categories separability. Due to the illumination and viewing directions reciprocity we used 3321 directions instead of the originally measured 6561 . Thus our dataset has 3321 features $\times 146$ (fabric), 16 (leather), 19 (wood) samples respectively. The number of classes was two or three, depending whether we computed features discriminating between two or all three tested categories. As a criterion function $J()$ identifying promising bidirectional features we used results of materials classification using linear SVM with one-level cross-validation. The SVM's penalty parameter $c$ was optimized on a validation dataset.

We typically evaluated about 300000 probes and the computational time was about 20 hours. Although this analysis is time-demanding it is performed once during off-line analysis of training data. Any further classification using the selected sparse features is very fast.

\section{Results and Discussion}

As a result of feature selection analysis we found out that only around ten bidirectional features (i.e., illumination/view directions) were enough to discriminate between all combination of classes with accuracy between $96 \%$ and 100\%. By the classification accuracy we denote ratio of correctly classified materials to all materials within the classified classes. Fig. 4 shows weights of the first ten DAF features illustrating the steepest decline for pairs wood-leather and wood-fabric that have also slightly better classification accuracy (shown in the graph legend). Therefore, even less features would be sufficient to accurately distinguish between our material categories.

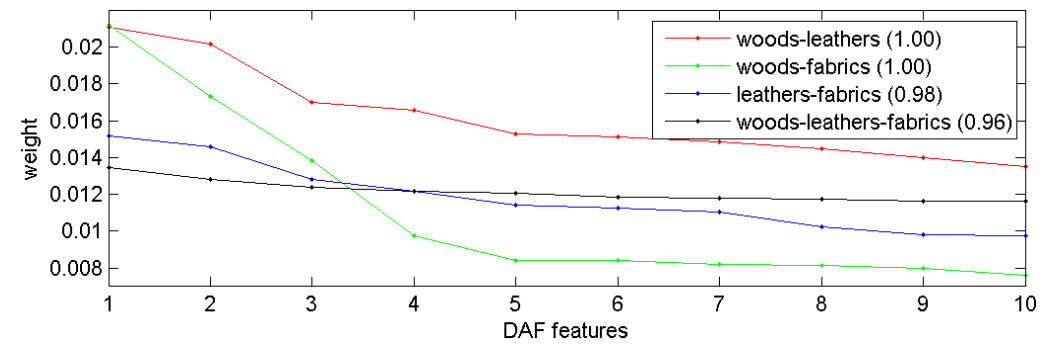

Fig. 4. Weights of the first ten DAF features (each representing a different combination of illumination and view directions) obtained for discrimination between individual categories of materials. The legend includes classification accuracy achieved using these ten features.

\footnotetext{
${ }^{4}$ http://fst.utia.cas.cz/
} 
wood - leather categories

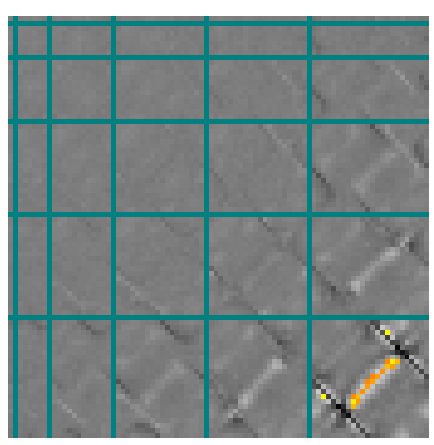

leather - fabric categories

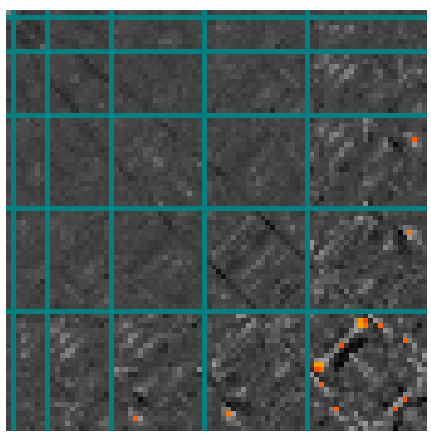

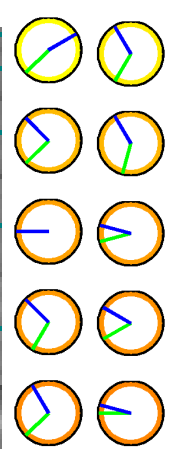

wood-leather-fabric categories

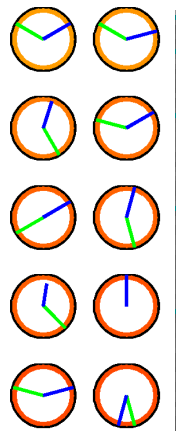

wood - fabric categories
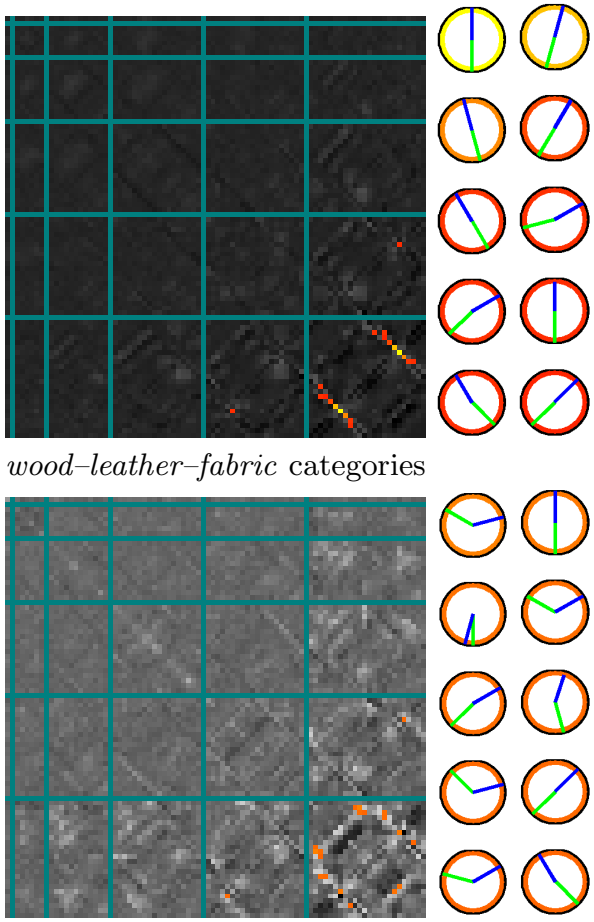

Fig. 5. Importance of individual directions for separability of several material groups. Each map represents 4D BRDF space unfolded into 2D image (Fig.1-right). Each pixel represents an unique combination of illumination and viewing directions and corresponding DAF features values are shown with brightness denoting their relative importance for recognition between the material groups. The ten most important directions are shown as color dots with color coding of DAF weights values from the highest (yellow) to the lowest (red). Right side of the images illustrates relation of corresponding 10 selected illumination (green) and view (blue) directions within hemisphere over a material. The remaining less important features are shown in gray.

Fig. 5 illustrates placement of DAF features in original 4D angular BRDF space unfolded to 2D image (see Fig.1-right). Majority of the important features are located in subspace of highest elevations (i.e., $75^{\circ}$ ), where typical BRDFs exhibit the highest contrast. The ten best features are shown in color coding from the most important (yellow) to less important (red). The right-hand side of the images show mutual positions of illumination (green) and view (blue) directions of the selected bidirectional features in the hemisphere viewed from the top. Color of the hemispheres border correspond to feature importance.

One can observe that classification between woods-fabrics categories relies on directions that lie directly on a specular highlight, i.e. view always opposite to illumination $\left|\varphi_{i}-\varphi_{v}\right| \approx \pi$. Note that the directions located near intersection of 


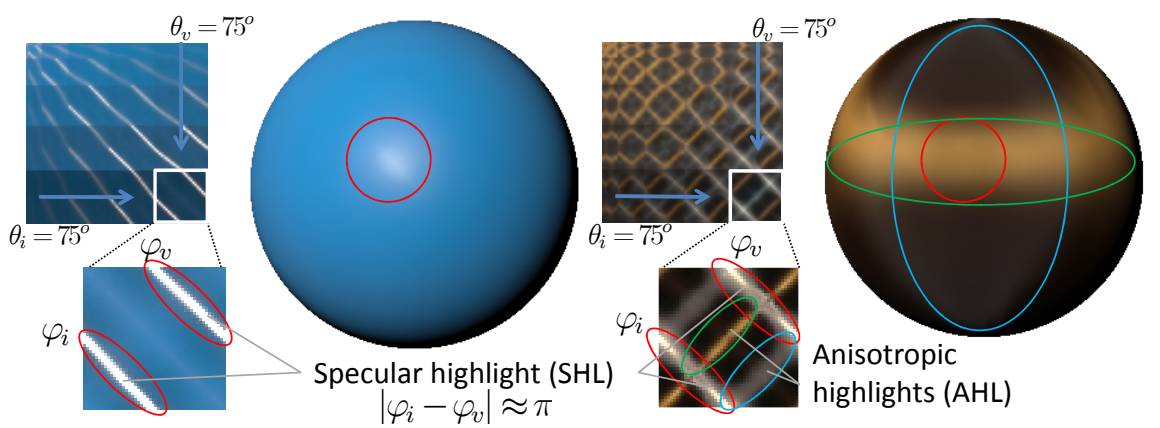

Fig. 6. Locations of specular and anisotropic highlights in the BRDF space and their appearance on a $3 \mathrm{D}$ object.

specular (SHL) and anisotropic (AHL) highlights (shown in Fig.6) have higher importance. This is due to a different typical behavior between these groups of materials.

In contrast, for wood-leather categories the selected features correspond to directions lying on location of main anisotropic highlight. This is intuitive as leather, in contrast to wood, does not exhibit any significant anisotropic highlight (including retro-reflection $\varphi_{i} \approx \varphi_{v}$ ). Again directions near intersections of SHL and AHL are deemed as more important.

For recognition between leather-fabric and wood-leather-fabric categories are the most important directions located near SHL and their primary function is detection of highlight's width. Therefore, the selected features lie on SHL and in its surrounding, as well as in retro reflective directions.

\subsection{Selected Features Stability Analysis}

Finally, we tested stability of the selected best features with regard to train dataset. We performed a two-level leave-one-out classification using linear SVM classifier between 17 wood and subset of 19 fabric having the most similar anisotropic properties as shown in Fig. 7 (BRDF subsets for fixed elevation angles $\left.\theta_{i} / \theta_{v}=75^{\circ} / 75^{\circ}\right)$. In each step, one material was removed from the test set the DAF features were computed on the remaining materials only.

Surprisingly, the DAF features obtained in each step were always identical. We have found that even with such a complex dataset, 12 directions (i.e., selected features) were enough to achieve an accurate classification of $97 \%$. When we performed the same experiment for wood, leather, and the subset of fabric (19) with 50 directions we achieved classification accuracy $92 \%$ and stability over $98 \%$, i.e., it suggests that about only $2 \%$ of directions changed over the cross validation. 


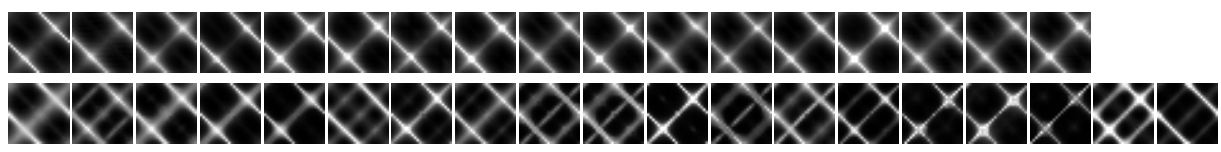

Fig. 7. Subspaces $\theta_{i} / \theta_{v}=75^{\circ} / 75^{\circ}$ of 17 wood (first row) and 19 selected fabric (second row) used in the stability experiment.

\subsection{Limitations and Future Work}

Although our test BRDF dataset provides a limited number of materials for each tested category, our cross validation classification experiment showed a promising performance of our method. We assume that using more materials within each category would further improve classification accuracy albeit possibly at the cost of a slightly increased number of selected features describing additional variability within a class of materials.

Another challenging issue is a computational time needed for the subset identification. Depending on the compared categories dimensionality, it took between two and six hours to find optimal discriminative directions using 24 cores $(3.3 \mathrm{GHz})$ of Intel Xeon E5-2643. However, once this subset is learned the speed of material classification using 10 features and linear SVM is negligible and the main bottleneck becomes acquisition of these ten directional samples for the unknown material to be classified. Performing FS off-line thus enables faster online performance, depending mainly on a speed of the reflectance measurements (e.g. photos) and their processing. Furthermore, our experiments revealed that majority of the selected features belong to the highest illumination and view elevation angles. Therefore, by neglecting low elevation angles we could potentially limit total number of features and thus effectively decrease computational time.

\section{Conclusions}

The objective of this paper was identification of very compact set of bidirectional gray-scale reflectance measurements that are capable of reliable classification between three different material categories (fabric, leather, wood). The point is to enable radical reduction of the cost of bidirectional features measurement and subsequent classification. We used dependency-aware feature selection technique for selection of sparse bidirectional features using BRDF database. We conclude that ten such gray-scale reflectance features are enough to keep classification accuracy $>95 \%$ on a BRDF database with moderate number of samples (evaluated by cross-validation). Furthermore, we have found that the stability of the selected bidirectional samples is very high, i.e., does not depend significantly on any particular material in the test dataset. Finally, we identified and discussed a proper locations of bidirectional samples for discrimination between our three material categories. Our initial results sufficiently demonstrate that feature selection helps to improve material classification methods relying on a very limited set of bidirectional samples. 


\section{Acknowledgments}

This research has been supported by the Czech Science Foundation grants 1402652S and 14-10911S.

\section{References}

1. Athanasakis, D., Shawe-Taylor, J., Fernandez-Reyes, D.: Learning non-linear feature maps. CoRR abs/1311.5636 (2013)

2. Filip, J., Vavra, R.: Template-based sampling of anisotropic BRDFs. Computer Graphics Forum 33(7), 91-99 (October 2014), http://staff.utia.cas.cz/filip/projects/14PG

3. Gu, J., Liu, C.: Discriminative illumination: Per-pixel classification of raw materials based on optimal projections of spectral BRDF. In: CVPR. pp. 797-804 (June 2012)

4. Haindl, M., Filip, J.: Visual Texture. Advances in Computer Vision and Pattern Recognition, Springer-Verlag, London (2013)

5. Jehle, M., Sommer, C., Jhne, B.: Learning of optimal illumination for material classification. In: Lecture Notes in Computer Science, vol. 6376, pp. 563-572 (2010)

6. Meister, G., Lucht, W., Rothkirch, A., Spitzer, H.: Large scale multispectral BRDF of an urban area. In: Geoscience and Remote Sensing Symposium, 1999. IGARSS'99 Proceedings. IEEE 1999 International. vol. 2, pp. 821-823. IEEE (1999)

7. Nicodemus, F., Richmond, J., Hsia, J., Ginsburg, I., Limperis, T.: Geometrical considerations and nomenclature for reflectance. NBS Monograph 160 pp. 1-52 (1977)

8. Qi, J., Kerr, Y., Moran, M., Weltz, M., Huete, A., Sorooshian, S., Bryant, R.: Leaf area index estimates using remotely sensed data and BRDF models in a semiarid region. Remote sensing of environment 73(1), 18-30 (2000)

9. Sandmeier, S., Deering, D.: Structure analysis and classification of boreal forests using airborne hyperspectral BRDF data from ASAS. Remote Sensing of Environment 69(3), 281-295 (1999)

10. Schaaf, C.B., Gao, F., Strahler, A.H., Lucht, W., Li, X., Tsang, T., Strugnell, N.C., Zhang, X., Jin, Y., Muller, J.P., et al.: First operational BRDF, albedo nadir reflectance products from MODIS. Remote sensing of Environment 83(1), 135-148 (2002)

11. Schaepman-Strub, G., Schaepman, M., Painter, T., Dangel, S., Martonchik, J.: Reflectance quantities in optical remote sensingdefinitions and case studies. Remote sensing of environment 103(1), 27-42 (2006)

12. Schick, E., Herbort, S., Grumpe, A., Wöhler, C.: Single view single light multispectral object segmentation. In: WSCG. pp. 171-178 (2013)

13. Somol, P., Grim, J., Pudil, P.: Fast dependency-aware feature selection in veryhigh-dimensional pattern recognition. In: Proceedings of the IEEE SCM. pp. 502509 (2011)

14. Wang, O., Gunawardane, P., Scher, S., Davis, J.: Material classification using BRDF slices. In: CVPR 2009. pp. 2805-2811. IEEE (2009)

15. Weinmann, M., Gall, J., Klein, R.: Material classification based on training data synthesized using a btf database. In: Computer Vision - ECCV 2014 - 13th European Conference, Zurich, Switzerland, September 6-12, 2014, Proceedings, Part III. pp. 156-171. Springer International Publishing (2014) 